\title{
SOME OBSERVATIONS ON THE EFFECT OF SENSORY STIMULATION UPON MINOR TREMORS IN MAN
}

\author{
Toshiyuki Ozaki, Kensuke Sato, Toshihiko AwazU, \\ Keiichi Mimura, Natsuo Honda, \\ Shigeyoshi Teramoto and Kazuo KitaJima* \\ The Second Department of Physiology, Nagasaki University \\ School of Medicine
}

\begin{abstract}
Recent reports by NAKAZAWA ${ }^{10)}$ and $\mathrm{KAWADA}^{6,5,7)}$ have described the results of some experiments concerning the effect of various sensory stimulations on the so-called minor tremors (MT) on the body surface of man which can not be observed by visual inspection. It was shown by NAKAzAwA that different changes are produced by flicker stimulation of $15 \mathrm{c} / \mathrm{s}$ in normal, neurotic and shizophrenic subjects while in the experiments by KAWADA, who studied the effect of various sonic stimulation on MT it was suggested that the increase of MT during sonic stimulation indicates the augmentation of muscle tonus. As pointed out by KAWADA, however, the physiological significance of the effects of various sensory stimulations on MT has not yet been clarified. In our previous investigation ${ }^{11}$, some relation had been demonstrated between MT and the electroencephalogram (EEG), which is known to represent the electrical activity of the brain. On the other hand, reports and discussions by several investigators ${ }^{1,2,3,4,15,16,17,18,19)}$ have shown that electroencephalographic responses caused by sensory stimulation may be led by gross electrodes from the sensory regions of the human brain. In the experiments of SATo et al. ${ }^{16}$, the frequency response of the brain led by gross electrodes was demonstrated to indicate the brain "activity", which is an extension of the concept of "excitability". Accordingly, it may well be deduced that changes corresponding to various sensory stimulations will be produced in MT as well as the EEG. The present study was carried out to elucidate the effect that may be produced in MT by rhythmic (or random) sensory stimulation, which is known to drive EEG responses of the same frequency as the stimulation.
\end{abstract}

Received for publication July 21, 1962.

* 尾崎俊行, 佐藤謙助, 粟津俊彦, 三村珪一, 本多夏生, 寺本成美, 北島和夫 


\section{METHODS}

The MT was led with a special pick-up (Nihon Kohden) from the thenar eminence of subjects relaxed in a dark, electromagnetically shielded room. At the same time, the electroencephalogram (EEG) from the occipital regions and the electrocardiogram (EKG) or heart beats were recorded. In some cases a correlogram was obtained by an analog correlator (Sony Co. Model CCA-22 Tokyo) for which the MT was recorded on magnetic tape by conversion of MT to electrical oscillations with the pick-up and amplified by the amplifier unit of an electroencephalograph (San'ei Sokki Co. Tokyo).

A photic stimulator (San'ei Sokki Co.) was used for delivering rhythmic or random photic stimulations to each subject. For random photic stimulation, random pulse signals recorded on magnetic tape were amplified to drive the photic stimulation. Rhythmic or irregular sonic stimulations, on the other hand, were applied by playing magnetic tapes on which rhythmic or random signals in the form of sine waves or pulses were recorded respectively. The former rhythmic sonic recording on magnetic tape was made by modulating the amplitude of the output oscillation from a high frequency oscillator with those of low frequency oscillator in sine form with a modulator.

In some cases, in addition to visual inspection of the MT tracings, the averageand frequency-patterns were obtained respectively from auto- and crosscorrelograms calculated by the simplified method of correlation analysis of SATO et a1.12,13,14) or an analog magnetic correlator and from KoBAYAshl's double harmonic analysis ${ }^{8,9}$ according to the same procedures as those used in the previous paper ${ }^{11}$.

\section{RESULTS}

In FIG. 1 and FIG. 2, the EEG, MT and EKG tracing recorded before and during rhythmic and random flash stimulations are illustrated respectively.

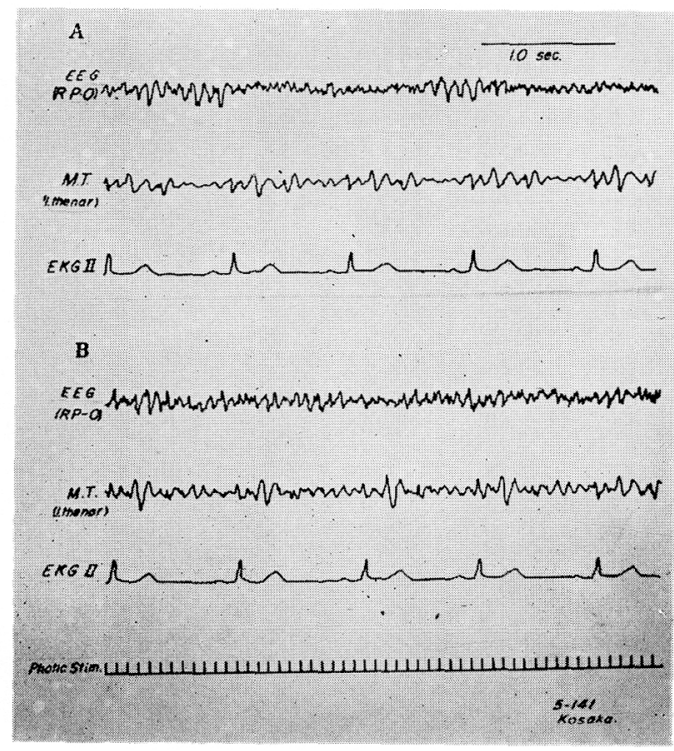

FIG. 1. EEG (occipital region), MT (1. thenar) and EKG (2nd lead) tracings before and during rhythmic flash stimulation of approximately $12 \mathrm{c} / \mathrm{s}$. A: Control EEG, MT and EKG before stimulation. B: EEG, MT, EKG and stimulation signals during stimulation. Note that the frequency of the EEG and MT responses is the same as that of the flash stimulation. 

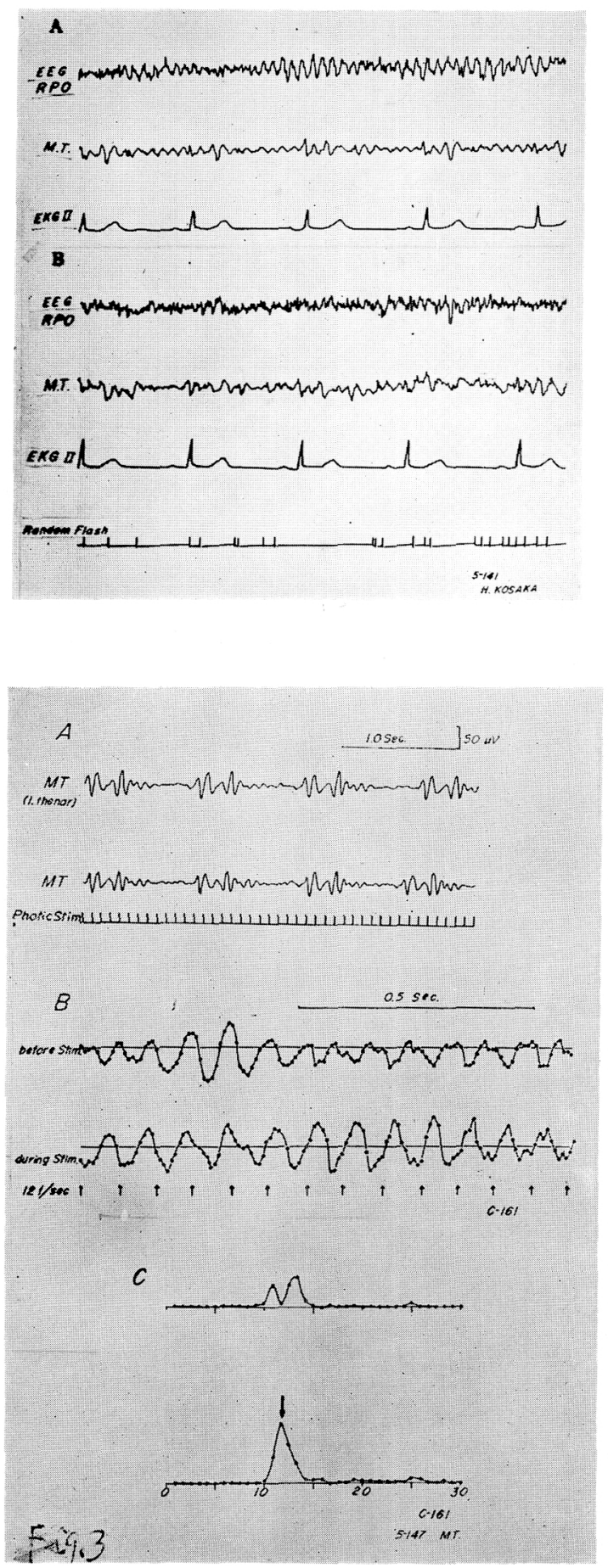

FIG. 3. MT tracings, crosscorrelograms and power spectra before and during rhythmic flash stimulation. A: above: Control MT tracing. below: MT and stimulation signals during stimulation. B: above: Crosscorrelogram between the rhythmic pulse of stimulating frequency and MT tracing before stimulation. below : Crosscorrelogram of stimulation and the MT tracing during stimulation. $\mathrm{C}$ : above: Power spectrum of crosscorrelogram (B: above) before stimulation. below: Power spectrum of crosscorrelogram (B: below) during stimulation. 
The EEG and MT in FIG. 1 show relatively regular components which synchronize with the rhythmic flash stimulations of $12 \mathrm{c} / \mathrm{s}$. The response caused by photic stimulation was more clearly observed in the EEG tracing than in the MT tracing, in which an increase in the amplitude of the dominant vibration and the appearance of fast components superimposed upon the driven oscillation were observed. In FIG. 2, however, irregular and fast components were found to be produced by random flash stimulation. Thus, not only the EEG but also the MT might possibly be driven regularly and randomly by rhythmic and random flash stimulations respectively.

In some cases in which the components driven by rhythmic flash stimulation were not recognized in the MT tracing but observed in the EEG tracing by visual inspection, driven MT components which synchronized with the stimulating frequency were demonstrated by the crosscorrelogram between the stimulation and MT. Shown in FIG. 3 is a typical case, in which the increase of the MT components, which synchronized with the stimulating frequency, was recognized in the crosscorrelogram between the stimulation and MT. Here,

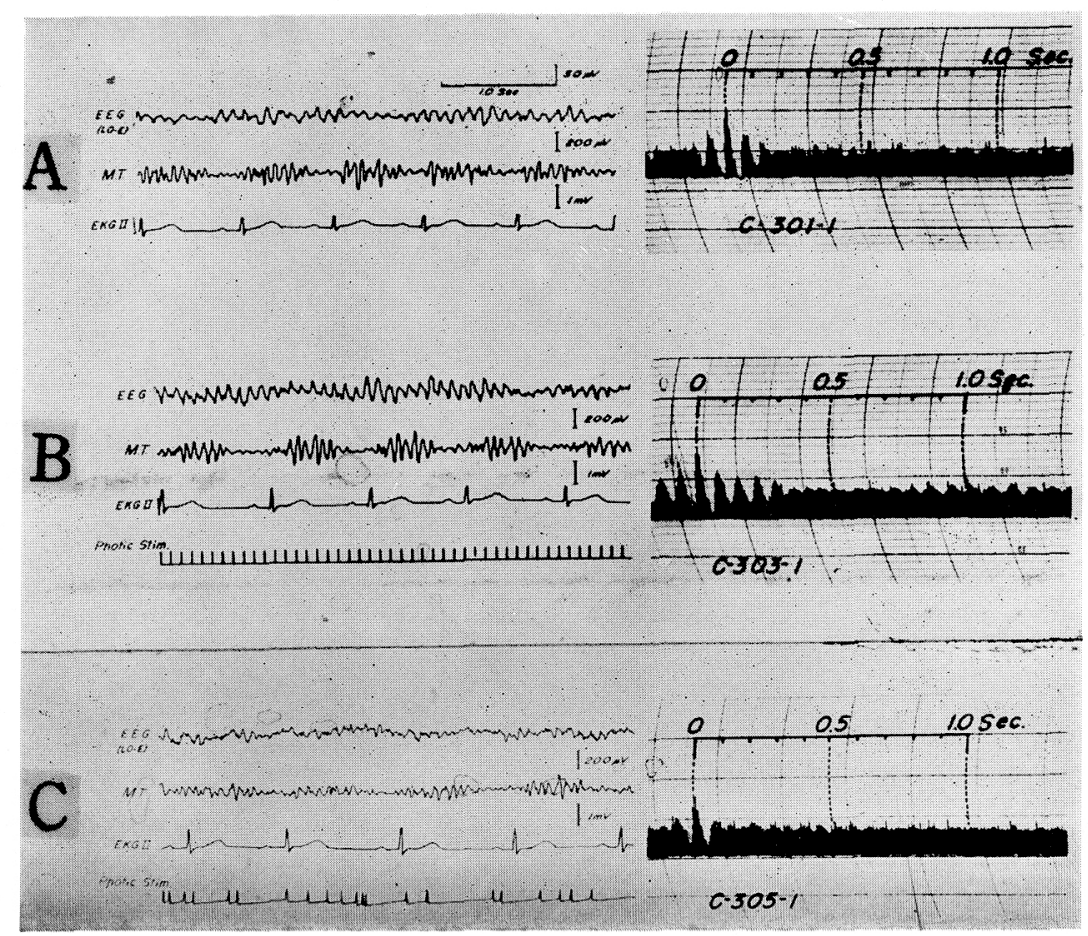

FIG. 4. EEG (occipital region), MT (1. thenar) and EEG (2nd lead) before and during rhythmic or random photic stimulation. A : Control before stimulation. left: EEG, MT and EKG. right: Autocorrelogram of MT. B: During rhythmic photic stimulation. $\mathrm{C}$ : During random photic stimulation. 
the crosscorrelogram between the rhythmic pulse set at the stimulating frequency and the MT tracing before stimulation was obtained as the control. In this crosscorrelogram, the components with the same frequency as the stimulating frequency will be picked up selectively in the control MT tracing. In the control crosscorrelogram, the amplitude of the oscillations is smaller than those during stimulation except in two swells. In the comparison of the power spectra of both crosscorrelograms, it was found that the spectrum of the control showed bimodal peaks located at frequencies slightly lower and higher than the stimulation frequency, while the spectrum during stimulation showed a monomodal peak corresponding to the stimulating frequency which indicates a synchronized response to the stimulation.

An increase of regularity and rhythmicity in the autocorrelogram was noted even in some cases, in which the appearance of components driven by rhythmic flash or sonic stimulation was not so clear in the MT tracings and its autocorrelograms obtained by analog correlator. In FIGs. 4 and 5 are shown representative EEG, MT and EKG tracings and the autocorrelogram of $\mathrm{MT}$ before and during photic or sonic stimulation. The former shows some effects

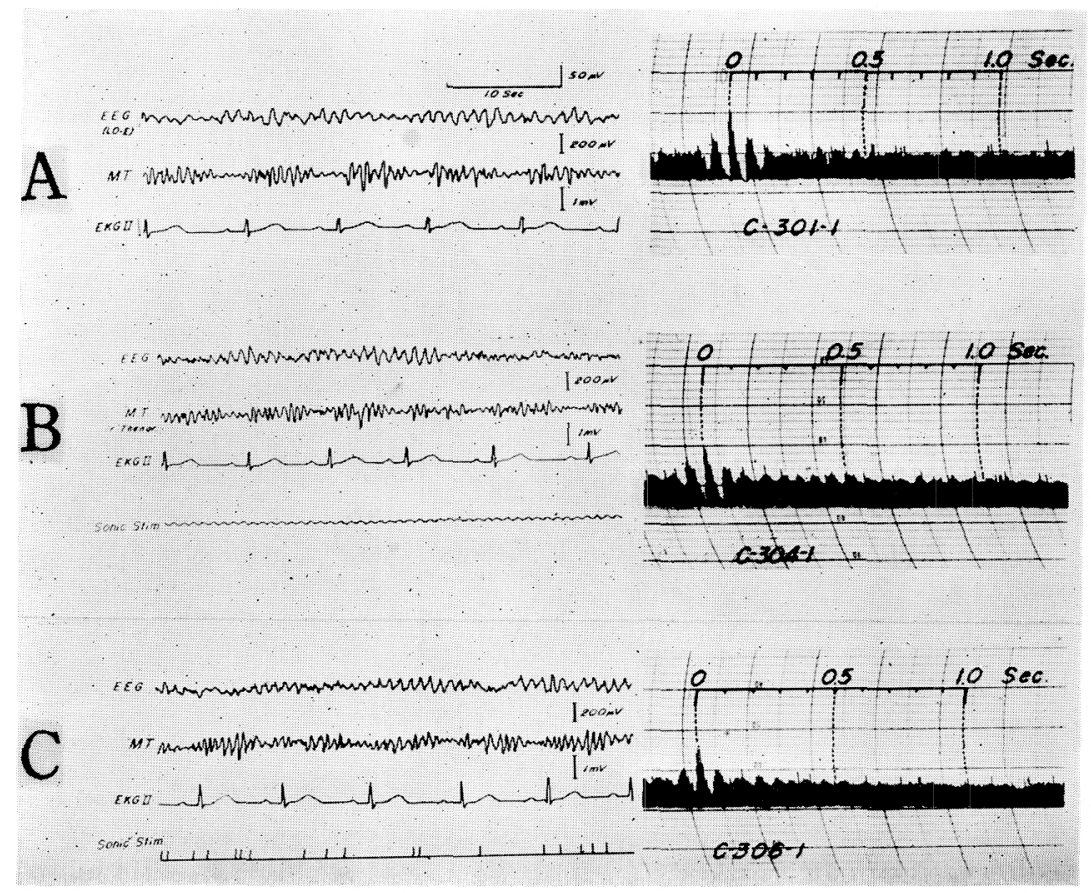

FIG. 5. EEG (occipital region), MT (1. thenar) and EKG (2nd lead) before and during rhythmic or random sonic stimulation. A: Control before stimulation. left: EEG, MT and EKG. right: Autocorrelogram of MT. B: During rhythmic sonic stimulation. C: During random sonic stimulation. 
of rhythmic and random photic stimulation on the EEG and MT. In the EEG tracing, a rhythmic stimulation was observed to produce driven EEG responses with a frequency the same as the stimulating frequency. In the MT tracing, however, it was difficult to observe any oscillations. which synchronize with the stimulation but an evident increase in the predominant vibration and some fast components which were not seen in the control tracing before stimulation did appear. By comparing the autocorrelograms of MT before and during stimulation, it is clear that the regularity, periodicity and amplitude of the vibrations are increased by the rhythmic stimulation. In contrast to this, random stimulation were shown to decrease periodicity and to increase damping of the predominant oscillation in the autocorrelogram. Similar effects of rhythmic and random sonic stimulations on the EEG and MT were also observed as illustrated in FIG. 5, though not so evident as with photic stimulation. The time interval between two successive $R$ waves of the EKG usually tended to become somewhat shorter with sensory stimulation such as rhythmic and random flash or sound.

In every MT tracing before and during rhythmic or random photic or sonic stimulation, the MT was found to correspond to the $R$ wave of EKG or heart beat as indicated in our previous paper ${ }^{11}$.

\section{DISCUSSION}

It was recently reported by NAKAZAWA ${ }^{10)}$ that normal, neurotic and schizophrenic subjects show different changes in MT over the frequency band of $8-13 \mathrm{c} / \mathrm{s}$ in response to flicker photic stimulation of $15 \mathrm{c} / \mathrm{s}$. In this experiment, the mean percentage of the alpha band decreased in 70 percent of neurotics and increased in 88 percent of the schizophrenic group respectively. In the normal group, the percentage of the alpha band tended to decrease somewhat though not so evident as in the neurotic group. In his investigation, however, no observation was carried out concerning the MT changes in response to the stimulation itself. In our experiments mentioned above, however, detailed studies showed that the EEG and MT responses to rhythmic and random flicker stimulations may be used as indicators of depression and augmentation of activities in the central and peripheral neuromuscular system. In some cases it was found that the driven MT responses were in synchronization with the frequency of the stimulation, although not so evident as in the EEG (FIG. 1). In addition, the autocorrelograms of MT revealed that increased periodicity and increased damping of the oscillation are produced respectively by rhythmic and random flicker stimulations.

As regards the otological aspects of MT, on the other hand, KAWADA ${ }^{6,5,7)}$, who observed the effect of the sonic stimulation on MT, has reported some data but he could not observe MT components corresponding to the stimulation 
itself. In his experiments, some changes in MT that demonstrate three phases were noted following such sonic stimulations as $1000 \mathrm{c} / \mathrm{s}$ pure tone, white noise and music (classic and symphonic). That is, immediately after the initiation of the stimulation, vibrations with a comparatively large amplitude continued for a short time after which there was a slow increase in amplitude of MT during the stimulation and immediately after cessation of the stimulation a slow increase of MT occurred. Consequently, the increase of MT during sonic stimulation was suggested to indicate the augmentation of muscle tonus. In our experiments, detailed observations were done on the effect of rhythmic and random stimulation on MT, that is the augmentation of periodicity and the increase of irregularity or the damping of oscillation corresponding to the former and latter stimulations respectively. However, such MT changes corresponding to each stimulation was not so evident as those in flicker stimulation. It seems likely that the appearance of such phenomena might be due to the change of muscle tonus due to such sensory stimulation as photic and sonic stimulations, as suggested by KAWADA and NAKAZAWA. The different responses in $\mathrm{MT}$ in these phenomena may perhaps be due to being driven regularly and irregularly by rhythmic and random sensory stimulation. It may have been very difficult, however, to detect these phenomena by simple visual inspection of MT tracing itself, because they are distorted and/or masked by superimposed irregular vibrations of various frequency, as has been indicated for the EEG tracing.

Many reports have been made on investigation concerning the appearance of driven EEG responses corresponding to rhythmic flicker and sonic stimulations. In our experiments it was clearly shown that rhythmic sensory stimulation caused changes in MT, though not so clear as those produced in EEG. On the other hand, the augmentation of damping in the oscillation of MT caused by random sensory stimulation, may correspond to the so-called arousal reaction that is produced by sensory stimulation in EEG tracing and which is well known to represent low amplitude and fast frequency. These findings seem to support the correlation between MT and EEG, as well as the various results presented in our previous paper ${ }^{11}$.

Therefore, from such corresponding changes in the EEG and MT responses that were observed in our experiments, it is suggested that sensory stimulation also plays an important role in the augmentation and inhibition of MT, which may provide insight into some aspects of the central mechanism of neuromuscular system.

\section{SUMMARY}

The effects of rhythmic or random photic and sonic stimulations upon the so-called minor tremors (MT) on the human body surface were studied. MT 
was traced by an electroencephalograph simultaneously with the recording of electroencephalogram (EEG) and heart beat or electrocardiogram (EKG). In addition to simple visual inspection of the tracings, the average time- and frequency-patterns were obtained for some cases by auto- and crosscorrelogram and power spectra analysis respectively.

The study of the MT tracing and crosscorrelogram between the stimulation and MT showed that MT components which synchronize with the frequency of the stimulation were driven by rhythmic flash stimulation. In addition, increased regularity and rhythmicity of MT were found in the autocorrelogram of MT, even when the driven component of MT produced by stimulation is not so evident on visual inspection. On the other hand, it was shown in the autocorrelogram that the damping of oscillation becomes faster by random flash stimulation. The same phenomena as those produced by photic stimulation were also demonstrated with rhythmic and random sonic stimulations, though is not so clear as those in rhythmic and random flash stimulations.

From the above results, it seems likely that the central process of the neuromuscular system such as augmentation and inhibition might be represented respectively by the increase and decrease of driven MT during various sensory stimulations, though ballistcardiographic oscillations are mixed in MT.

\section{REFERENCES}

1) Adrian, E. D. and Matthews, B.H.C. The Berger rhythm: potential changes from the occipital lobes in man. Brain, 57:355-384, 1934.

2) Brazier, M. A.B. and CAsby, J.U. A study of the EEG in normal subjects during intermittent photic stimulation. Electroceph. clin. Neurophysiol., 3: 380-381, 1951.

3) BARLow, J.S. Rhythmic activity induced by photic stimulation in relation to intrinsic alpha activity of the brain in man. Electroenceph. clin. Neurophysiol., 12: 317-326, 1960.

4) Davis, P. A. Effects of acoustic stimuli on the waking human brain. J. Neurophysiol., 2 : 494-499, 1939.

5) *Kawada, M., Takesue, M. and Nishinara, H. Audiology, 3: 26, 1960.

6) *Kawada, M., Tsuji, M., Takesue, M. and Inanaga, K. Audiology. 1: 236, 1958.

7) *KAwAdA, M. Some otological aspects of the microvibration and its findings. Otologia Fukuoka, $7:$ 124, 1961.

8) *Kobayashi, T. On the multi-harmonic analysis (I). Jour. Met. Soc. Jap. II. 31 : 370-389, 1953.

9) Kobayashi, T. On the multi-harmonic analysis (II). Jour. Met. Soc. Japan Series II. $33: 68-75,1955$.

10) *Nakazawa, Y. Clinical application of MT test with photic flicker stimulation. Psychiatr. Neurol. Jap. 62 : 810, 1960.

11) Ozaki, T., Sato, K., Awazu, T., Mimura, K., Honda, N., Teramoto, S. and KitaJIMA, K. Some observations on minor tremors related to heart beat. Jap. J. Physiol., 12 : 484-493, 1962.

12) Sato, K., Honda, N., Mimura, K., Ozaki, T., Teramoto, S. and Kitajima, K. 
A simplified method for determining the average response time-pattern of the evoked potential in electroencephalgraphy. Electroenceph. clin. Neurophysiol., 14 : 764-766, 1962.

13) Sato, K., Honda, N., Mimura, K., Ozaki, T., Masuya, S., Teramoto, S. and Kitajima, K. A simplified method for crosscorrelation analysis. Electroenceph. clin. Neurophysiol., $14: 767-768,1962$.

14) Sato, K., Honda, N., Mimura, K., Ozaki, T., Teramoto, S., Kitajima, K. and Masuya, S. A simplified method for autocorrelation analysis in electroencephalography. Electroenceph. clin. Neurophysiol., 14: 769-771, 1962.

15) Sato, K., Ozaki, T., Mimura, K., Masuya, S. and Honda, N. On the frequency response of EEG activity obtained by the intermittent click stimulation. Electroenceph. clin. Neurophysiol. Suppl. $18: 5-7,1959$.

16) Sato, K., Ozaki, T., Mimura, K., Masuya, S., Honda, N., Nishikawa, T. and SonodA, $T$. On the physiological significance of the average time- and frequencypatterns of the electroencephalogram. Electroenceph. clin. Neurophysiol., 13: 208215, 1961.

17) Toman, J. Flicker potentials and the alpha rhythm in man. J. Neurophysiol. 4 : 57-61, 1941.

18) Walter, W. G. Dovey, V. J. and Shipton, H. Analysis of the electrical response of the human cortex to photic stimulation. Nature, $158: 540-541,1946$.

19) WAlter, V.J. and WALter, W. G. The central effects of rhythmic sensory stimulation. Electroenceph. clin. Neurophysiol., 1 : 57-86, 1949.

* Written in Japanese. 